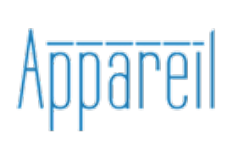

Appareil

12 | 2013

Walter Benjamin. Politiques de l'image

\title{
Un cinéma des passages - les fantasmagories du cinéma de Jacques Demy
}

\section{Alain Naze}

\section{OpenEdition}

1 Journals

Édition électronique

URL : http://journals.openedition.org/appareil/1549

DOI : 10.4000/appareil. 1549

ISSN : 2101-0714

Éditeur

MSH Paris Nord

Référence électronique

Alain Naze, "Un cinéma des passages - les fantasmagories du cinéma de Jacques Demy », Appareil [En ligne], 12 | 2013, mis en ligne le 27 mars 2013, consulté le 30 juillet 2020. URL : http:// journals.openedition.org/appareil/1549; DOI : https://doi.org/10.4000/appareil.1549

Ce document a été généré automatiquement le 30 juillet 2020.

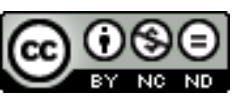

Appareil est mis à disposition selon les termes de la Licence Creative Commons Attribution - Pas d'Utilisation Commerciale - Pas de Modification 4.0 International. 


\title{
Un cinéma des passages - les fantasmagories du cinéma de Jacques Demy
}

\author{
Alain Naze
}

1 L'idée d'une mise en écho du cinéma de Jacques Demy et de l'œuvre de Walter Benjamin (mise en écho qui pourrait d'ailleurs être prolongée à plusieurs niveaux, notamment en ce qui concerne une certaine conception du bonheur, comme «remplissement rétrospectif d'un vœu $»^{2}$ ) m'est venue d'abord de la considération de la place prépondérante de certains lieux privilégiés du livre inachevé de Benjamin (Paris, capitale du XIX $x^{e}$ siècle) dans le cinéma de Demy : le passage bien sûr (en l'occurrence le passage Pommeraye, à Nantes, qu'on retrouvera dans Lola, Les Parapluies de Cherbourg, ou encore dans Une chambre en ville), mais aussi le casino (d'Enghien, de Nice, puis de Monte-Carlo, dans La Baie des Anges), ou encore le pont transbordeur, cette architecture métallique, présente de façon récurrente, dans Les Demoiselles de Rochefort, et artificiellement reconstituée sur un fond d'illusion d'optique dans Une chambre en ville, pour Nantes, ou encore dans Trois places pour le 26, pour la ville de Marseille cette fois (le pont étant présenté alors comme un élément de décor du spectacle de Montand).

2 Dès lors, la question qui a commencé à prendre forme était celle de l'articulation entre architecture et cinéma, ou, pour être plus précis, celle de l'articulation entre trois niveaux de perception: la perception face à une architecture, la perception face à un film, et enfin la perception face à un film mettant le spectateur en présence d'une architecture. L'idée d'un englobement d'une forme de perception (et donc aussi de temporalité) dans une autre m'intéressait ici particulièrement, puisqu'on sait que Benjamin apparente les modalités de perception en présence d'une architecture à celles qu'on éprouve en présence d'une production cinématographique, établissant qu'elles relèvent, les unes comme les autres, d'une forme de perception distraite :

[...] celui qui se recueille devant une œuvre d'art s'y abîme ; il y pénètre comme ce peintre chinois dont la légende raconte que, contemplant son tableau achevé, il y disparut. Au contraire, la masse distraite recueille l'œuvre d'art en elle. Les édifices en sont les exemples les plus évidents. De tout temps, l'architecture a été le 
prototype d'une œuvre d'art perçue de façon à la fois distraite et collective. [...] La réception par la distraction, de plus en plus sensible aujourd'hui dans tous les domaines de l'art, et symptôme elle-même d'importantes mutations de la perception, a trouvé dans le cinéma l'instrument qui se prête le mieux à son exercice ${ }^{3}$.

Dès lors, c'est l'enveloppement de l'espace urbain, particulièrement sous la forme architecturale des passages, au sein d'un appareillage cinématographique producteur d'images qui est à interroger. En effet, de même qu'à l'identité de structure entre le passage et le panorama, Jean-Louis Déotte ajoutait cet écart par lequel «le panorama apporte une dimension de réflexivité que ne possède pas le passage, malgré ses alignements de miroirs", réflexivité faisant du panorama une véritable "monade leibnizienne ", de même, donc, le cinéma apporterait " une dimension réflexive que les appareils strictement urbains n'auraient pas en eux-mêmes $»^{4}$. Il s'ensuit que les interprétations multiples de la ville, selon des appareillages divers et non strictement urbains, renvoient une image démultipliée de la ville, selon des perspectives donnant l'impression d'une multiplicité effective d'univers, quand ce sont les points de vue qui varient. À cette description leibnizienne de la ville, on peut ajouter, en suivant JeanLouis Déotte là encore, que ce serait moins la ville qu'il s'agirait de cerner (en cette objectivité qu'elle conserverait, par-delà les perspectives, dans le schéma leibnizien), que « l'appareil psychique » lui-même ${ }^{5}$ : c'est qu'à l'écart d'une perception volontaire et attentive, architecture et cinéma se caractérisent par une perception non focalisante et tactile, nous orientant plutôt vers la mémoire involontaire proustienne, raison pour laquelle spectateur de cinéma et flâneur peuvent légitimement être apparentés. En ce sens, c'est évidemment bien moins une reconstitution fidèle de la ville de Nantes du début des années cinquante qui conduit Jacques Demy à réintégrer le pont transbordeur au décor d'Une chambre en ville (1982) - c'est bien plutôt la mémoire corporelle, tactile qu'il conserve de cette ville de Nantes d'avant le démontage du pont transbordeur en 1958, qui l'amène à recourir à ce trucage cinématographique, le glassshot (« on interpose entre la caméra et le décor [...] une plaque transparente sur laquelle est peint un élément du décor qui, à l'image, masquera ce qui se trouve derrière sa surface opaque et s'intégrera au milieu de ce qui est visible à travers la plaque $»^{6}$ ). Par ce procédé, l'Autrefois se révèle n'être pas seulement du passé (ce recours au passé révolu qui limite l'intérêt de toute reconstitution), mais bien de l' actuel - ce fragment urbain, isolé, détaché de la Nantes réelle de 1950 (au point de réapparaitre, comme type d'architecture, à Rochefort et à Marseille) entrera en composition notamment avec cet autre fragment qu'est le passage Pommeraye, Une chambre en ville réalisant à cet égard une construction à partir du montage d'éléments fragmentaires, à l'image du travail d'un historien chiffonnier. Il ne s'agira évidemment pas de confondre ces deux types de fragments, c'est-à-dire ceux qui relèvent de la construction d'un décor cinématographique (les éléments de la ville repeints pour l'occasion, par exemple, mais aussi le trucage aboutissant à l'image du pont transbordeur dans Une chambre en ville), avec les éléments architecturaux utilisés sans transformation, comme le passage Pommeraye - absence de modification qui, seule, rend possible ce fait, souligné par Suzanne Liandrat-Guigues à propos du cinéma de Demy, que, «[d]e film en film, le passage Pommeraye se donne pleinement à voir pour lui-même $»^{7}$. Cette précision est d'importance, puisqu'elle permet d'ouvrir la réflexion sur les observations de celui dont on peut considérer qu'il développe, dans le domaine du cinéma, la théorie que Walter Benjamin n'aura pas eu le temps de concrétiser, Kracauer, qui écrivait en effet : « Le film est pleinement lui-même lorsqu'il enregistre et 
révèle la réalité matérielle. Or cette réalité comporte nombre de phénomènes qui ne sont guère perceptibles que grâce à la capacité qu'a la caméra de les saisir au vol. Et comme tout médium privilégie les choses qu'il est seul à pouvoir rendre, on peut s'attendre à ce que le cinéma soit mû par le désir de représenter la vie matérielle en ce qu'elle a de fugitif, de plus éphémère. Les foules des rues, les gestes involontaires, les impressions passagères sont ses mets de choix. [...] Je postule donc que les films sont fidèles au médium dans la mesure où ils explorent le monde que nous avons sous les yeux $"^{8}$. Il faudra bien sûr accorder toute son importance à l'idée selon laquelle le cinéma est capable de nous révéler ce qu'on a sous les yeux, et donc pas seulement de nous le restituer, ce qui enfermerait en effet le cinéma dans un cadre strictement positiviste (si le cinéma restituait la matérialité du monde, simplement, cela laisserait entendre que cette matérialité a déjà été saisie en dehors du médium cinématographique, ce qui réduirait ce dernier au statut de simple véhicule).

4 Voyons donc à présent ce qu'il en est de cette fidélité au médium dans le cinéma de Jacques Demy, ou plus précisément, comment les films du réalisateur nantais se caractérisent par une puissance de révélation cinématographique de la réalité matérielle, m'apparaissant inséparable de la joie provoquée par les films de Demy - et pas seulement une joie un peu triste parce qu'il en irait peut-être ici d'une nostalgie qui me serait propre, et qui me reconduirait à mes souvenirs d'enfance, effectivement nantaise. Non, c'est une couche beaucoup plus profonde de l'enfance qui est ainsi affectée.

5 Faisons d'abord remarquer que si le flâneur des passages et le spectateur de cinéma sont bel et bien apparentés, c'est pour leur commune exposition à un univers de fantasmagories - l'un et l'autre ont affaire à un appareillage pourvoyeur d'images oniriques. Par ailleurs, l'univers de rêve qui leur est commun est celui du rêve collectif, celui de la masse, percevant de façon distraite, et non l'univers du rêveur singulier, dont la perception reste contemplative. Dans ces conditions, les choses perçues dans les domaines de l'architecture et du cinéma n'atteignent pas la claire conscience propre à la connaissance, mais sont absorbées corporellement, ce qui empêche d'opposer la veille et le rêve, et donc interdit de faire du réveil une prise de conscience. C'est ce qu'établit Jean-Louis Déotte, lorsqu'il fait remarquer que le réveil a lieu «dans une topologie où l'enveloppe n'est que la forme extérieure de ce qu'elle enveloppe, la forme enfermant le contenu comme dans l'exemple de "la chaussette en dedans". Ce n'est plus le règne de la répétition du désir ou de l'ennui, mais celui de l'événement provoqué par une remémoration [...] »9. Entre l'appareil des passages et l'appareil cinématographique, il y a donc ce point commun qu'en eux veille et rêve se distinguent seulement comme intériorité et extériorité, le réveil s'effectuant à travers la remémoration, qui est simplement la manifestation de la trace mnésique inconsciente, à ne pas confondre avec le perçu lui-même - la remémoration faisant revenir ce que jamais on n'a vécu, c'est-à-dire cette part inaperçue de notre vécu. Dans ces conditions, qu'on parle du monde rêvé du cinéma de Demy, cela n'en fait aucunement, en soi, un cinéma qui s'opposerait au réveil du public, contre les formes cinématographiques plus visiblement engagées, politiquement - que le film Une chambre en ville, par exemple, soit un film politique, cela tient d'ailleurs sans doute moins au propos qui est le sien qu'à l'actualisation spécifique de l'archive qu'il effectue en provoquant la remémoration d'une révolte ouvrière, notamment en recourant à la forme fictionnelle du mélodrame, comme Fassbinder lui-même, d'ailleurs, n'hésitera pas à le faire. 


\section{De Ophuls à Demy}

$\mathrm{Si}$, avec son livre sur les passages parisiens, Benjamin analyse bien l'appareil des passages, on peut dire que l'analyse du cinéma comme appareil reviendra plutôt à Kracauer lui-même, on l'a dit, étant entendu que chaque époque étant configurée selon un certain âge des appareils, celui qui triomphe à l'époque de Benjamin (le cinéma) conserve pour lui un certain caractère nouménal, le rendant imparfaitement connaissable. De ce point de vue, Max Ophuls (dont on sait qu'il est du nombre des cinéastes qui ont donné à Demy le désir de faire du cinéma, à côté de Bresson, notamment) serait, dans une certaine mesure, vis-à-vis de Jacques Demy, comme Benjamin par rapport à Kracauer, comme le montre le cas du film La Ronde (1950), par exemple. En effet, Ophuls y met bien en scène la ville de Vienne (comme dans Liebelei, De Mayerling à Sarajevo et Lettre d'une inconnue), filmée cinq ans après qu'elle ait été libérée du régime nazi, et, comme le souligne le théoricien du cinéma et spécialiste de l'histoire des médias Karl Sierek, «les sculptures de la Michaelerplatz de Vienne, devant la tour principale du château, et la coupole du musée de l'Histoire de l'Art resplendissent - face au luxe détonant des bâtiments de la spéculation fin-de-siècle et des boulevards extérieurs... Tout cela s'inscrit dans le système de représentations du "film viennois", avec un champ temporel qui s'étend du congrès de Vienne jusqu'au début de la première guerre mondiale, même si Ophuls ne fait pas de films "de genre" dans le sens classique du terme. Avec lui, les décors baroques ou la mode Biedermeyer, les uniformes fantaisistes et les costumes stylisés, les célébrités de l'art, de la politique ou de la hiérarchie militaire apparaissent en marge, voire en surface. Les images à référence historique se détachent de leur référent, à rebours des films de Willi Forst, d'Ernst Marischka ou de Walter Reich, et jouent librement leur rôle d'allégories de la modernité $»^{10}$. Autrement dit, la Vienne d'Ophuls emprunte réellement à la Vienne historique, mais l'intention du cinéaste n'est aucunement de reconstitution, la ville qu'il met en scène visant surtout à faire apparaître " un ailleurs par-delà les attaques auxquelles il a dû faire face, Juif dans la Vienne du début des années vingt $»^{11}$. Il y a donc une volonté de déréalisation de la ville, chez Ophuls, qui le conduit notamment, dans $L a$ Ronde, à la nimber d'une brume qui, estompant ses contours, apparente la ville à un décor de rêve. Chez Demy, on retrouvera cette tendance à la déréalisation, notamment lorsqu'il recourra aux peintures vives pour la ville de Cherbourg, par exemple, mais aussi lorsqu'il repeindra en bleu le marché du Bouffay, à Nantes, ou encore lorsqu'il voudra que tous les dialogues du film soient chantés (Les Parapluies de Cherbourg, Une chambre en ville) indiquant ainsi ostensiblement qu'on est dans l'univers de la fiction, voire du rêve. Pourtant, comme on va le voir, il y aura aussi, à la différence de ce qui se passe chez Ophuls, des éléments de réalité matérielle non transformés en décor cinématographique, qui résisteront chez Demy, et l'on pourrait même dire qu'ils s'affirmeront d'autant mieux en cette matérialité que les autres éléments du film, lors même qu'ils seraient référentiels (et les villes de Nantes, ou de Cherbourg par exemple le sont en effet), ne recevront pas un traitement réaliste. Et puis surtout, Ophuls semble vouloir mettre en scène l'opération cinématographique elle-même, comme pour la démasquer, en ne maintenant qu'artificiellement la fiction: «Je suis l'incarnation de votre désir. [...] Mais où sommes-nous ici ? Sur scène ? Dans un studio ? [...] Ah... Nous sommes à Vienne. Dix-neuf cents. Changeons de costume. Dix-neuf cents. Nous sommes dans le passé. J'adore le passé. C'est tellement plus reposant que le présent - et 
tellement plus sûr que l'avenir ", déclare le meneur de jeu, le responsable de la ronde, autant dire le cinéaste. Il dénude ainsi les mécanismes de la fiction, faisant par conséquent de la Vienne mise en scène une ville du passé, c'est-à-dire une scène où tout s'est déjà joué, où rien d'inattendu ne peut plus surgir. En cela, le meneur de jeu nous enferme dans la répétition, l'ennui, d'où ne pourra surgir aucune nouveauté. Ophuls réalise ainsi une pure forme, qui, si elle procure bien un plaisir au spectateur, ne le fait qu'à partir de la matière cinématographique elle-même, et donc pas à partir du "monde que nous avons sous les yeux ", selon la formule de Kracauer. Qu'à côté de la scène de théâtre, Ophuls ait également donné à voir une caméra de cinéma, cela n'empêche pourtant pas que ce dispositif nous maintienne hors d'une possibilité de réveil : la prise de conscience du dispositif fictionnel ne peut nous faire sortir de la ronde de l'éternel retour du même, de l'ennui, précisément parce qu'en procédant ainsi, on reconduit la coupure rêve/veille, ce qui prive les éléments matériels de la réalité viennoise de toute possibilité de provoquer le moindre processus de remémoration - il faudrait qu'on ait sauvegardé la possibilité d'un surgissement du passé, ce que le préambule empêche précisément, en dévitalisant le passé, le présentant comme révolu, et par là même rassurant, puisque incapable de produire la moindre surprise, incapable de détenir encore la moindre virtualité de surgissement d'un événement. Que le mouvement de la ronde puisse être mis à mal dans sa répétition à l'identique, cela ne pourra plus désormais se jouer que dans l'espace de la fiction, la matière de la réalité viennoise ayant été mise hors circuit une fois pour toutes. La "panne" sexuelle d'Alfred (Daniel Gélin) provoquera seulement la panne mécanique (et momentanée) du manège, c'est-à-dire de la ronde - c'est donc de l'intérieur même de sa structure (et seulement à partir de cet intérieur) que la forme construite par Ophuls sécrète les possibilités de son propre enraiement. Ophuls tendrait donc à nous enfermer dans la fantasmagorie, en transformant l'histoire en nature si l'on veut, c'est-à-dire en utilisant les innovations techniques pour les transfigurer en images oniriques. En cela, Karl Sierek a raison de soutenir, comme on l'a vu, que Max Ophuls utilise les références historiques comme des allégories de la modernité - les passages eux-mêmes avaient cette fonction allégorique, consistant à détacher les choses de leurs corrélations, afin de les exposer comme marchandises. Sous ce rapport, le livre de Benjamin se voulait " une allégorie de l'allégorie "12, dans le sens où il s'agissait d'isoler certains objets d'étude, selon la technique du montage, de manière à briser ces objets (en les conduisant à l'état de ruines, en les mortifiant à travers leur séparation d'avec les " corrélations ordinaires de la vie $»^{13}$ ), mais aussi de façon à les conserver (à l'état de ruines, objets ainsi conduits à leur signification). Si la technique d'Ophuls consistant à détacher la Vienne filmée de la Vienne historique obéit bien à l'idée, propre à l'allégoriste, de briser l'objet, en le conduisant à l'état de ruine, il lui manque en revanche l'autre dimension, qui aurait permis à Ophuls de réaliser une allégorie de l'allégorie, c'est-à-dire de conserver l'objet à l'état de ruine, car il détruit purement et simplement l'objet, en l'inscrivant dans un passé révolu. La question qu'on peut se poser à présent, qu'on peut enfin énoncer serait donc celle consistant à se demander si le cinéma de Jacques Demy parvient effectivement à réaliser une allégorie de l'allégorie, c'est-à-dire si, en filmant la réalité matérielle, il parvient à lui conserver cette capacité à provoquer le phénomène de la remémoration. 


\section{Lola (1961) - première tentative d'approche cinématographique du passage}

7 Indiquons tout de suite que si le passage Pommeraye, à Nantes, constitue l'objet à partir duquel la réflexion autour de Jacques Demy et sa capacité à faire que son cinéma se révèle "allégorie de l'allégorie " se pose, c'est que ce passage ne subira aucune transformation, qui aurait visé à en faire un élément de décor. C'est la matérialité du passage qui s'imposera donc, de manière récurrente, à l'écran, c'est-à-dire la matérialité non immédiatement donnée, mais telle que le cinéma peut la révéler. Et d'abord dans Lola, film de 1961, dédié à Max Ophuls, et matrice de toute la filmographie de Jacques Demy.

8 La double nature des passages (à la fois productrice d'ennui à travers l'étalage des marchandises, et le phénomène de la mode, mais aussi productrice de nouvelles démarches, de nouvelles postures) est indiquée à travers le fait que Roland Cassard, se plaignant depuis le début du film de s'ennuyer, au point de rêver pour conjurer cette langueur (au travail, il répond à son patron lui reprochant de "bayer aux corneilles »: "Je rêve, tout au plus"; au cinéma nantais le Katorza, il va voir Retour au paradis, avec Gary Cooper, à seule fin de s'évader), finit par accepter l'idée de se rendre au passage Pommeraye pour un rendez-vous susceptible d'ouvrir sur un voyage, et là, rencontre fortuitement Lola/Cécile (qu'il avait connue autrefois) au rez-de-chaussée du Passage. La déambulation ennuyée de Roland, au début du film, relève bien du personnage du flâneur que les passages vont engendrer, et qu'il flâne alors dans la rue importe peu; quant à la rencontre imprévue dans le passage, elle correspond aux rêves qui circulent dans ces boyaux collectifs : «Le passage n'est que la rue lascive du commerce, propre seulement à éveiller les désirs ${ }^{14}$, écrit Benjamin. Sous ce rapport, le personnage de Lola est significatif en son ambiguïté même, comme le souligne Suzanne LiandratGuigues : « Lola (appelée en réalité Cécile) est une danseuse de cabaret. [...] Dans le film, Lola a enfilé, à la hâte, un simple imperméable sur sa guêpière de scène, pour aller chercher son fils. Ce détail vestimentaire ambigu se combine au jeu de l'actrice pour tenir lieu de portrait d'une adorable péripatéticienne $»^{15}$. Au personnage du flâneur vient donc se joindre celui de la prostituée (ce que n'est pas Lola, malgré bien des traits l'apparentant à une prostituée - lorsque Roland jugera qu'elle l'a trompé avec un marin, il la traitera d'ailleurs de "putain»). Roland va tomber amoureux de Lola, ce que, dans l'espace du passage, on peut interpréter comme une forme de fétichisation de la marchandise (même si Benjamin insiste bien pour ne pas assimiler la prostituée à la marchandise - elle n'en est que l'allégorie, étant aussi la vendeuse). L'aura qui entoure Lola dans les yeux de Roland (et le processus de fétichisation de la marchandise serait bien l'effet de " la masse concentrée dans les passages $~^{16}$ ) entraînerait alors ce dernier dans une fantasmagorie, une ivresse le sortant de son ennui, mais le plaçant dans un rapport de dépendance à l'égard du fétiche.

On pourrait également insister sur la composition en miroir de tout le film, qui renvoie aux miroirs des passages eux-mêmes (comme celui dans lequel Yvon, le fils de Lola, joue à se regarder), ou encore à l'ennui et à la mélancolie liée à la répétition du même, mais aussi à la structure monadique du film: l'histoire de Lola est en quelque façon répétée par celle de Cécile Desnoyers, en accéléré (court laps de temps dans la vie de cette Cécile, qui a 14 ans), mais aussi au ralenti (cinématographiquement, la scène filmée au ralenti entre elle et Frankie, au manège, à l'occasion de son quatorzième 
anniversaire - ce chiffre 14 renvoyant lui-même au Katorza, cinéma nantais où, adolescent, Demy se rendait fréquemment); la crainte de $\mathrm{M}^{\mathrm{me}}$ Desnoyers est, de son côté, que sa fille répète son histoire, et devienne mère célibataire - et cette répétition se configure, lorsque Cécile, à la fin du film, part pour Cherbourg (ville en laquelle sa mère s'est retrouvée enceinte), rejoindre son oncle coiffeur, qui est en fait son père ; etc. Cette structure monadique pourrait d'ailleurs, dans une certaine mesure, être étendue à l'ensemble du cinéma de Demy, avec un grand nombre de personnages récurrents, ou de situations se répétant de film en film. Mais c'est aussi l'architecture ornementale du passage Pommeraye qui va elle-même venir participer à ce jeu de miroir, en redoublant la profusion des marchandises, comme on le voit dans le cadre de la dernière rencontre entre Lola et Roland, celle-ci l'ayant entraîné vers le dernier niveau, appelé « galerie des statues », ou « galerie de l'Horloge »:

[...] le Passage est caractérisé par une profusion d'ornements architecturaux venant dédoubler, en un miroitement spécifique, l'abondance des marchandises et des objets exposés dans ce haut lieu du commerce en tout genre ${ }^{17}$.

On retrouve ici, dans la façon même dont le passage est filmé, en sa matérialité, l'idée de Benjamin selon laquelle, dans les passages, l'armature de fer est masquée, refoulée par une façade boursouflée - c'est ainsi la technique qui est refoulée, en son potentiel de rêve collectif, au profit d'une apparence artistique, destinée aux rêves privés des particuliers. Mais ce sont aussi les mouvements de caméra, autour de Lola et Roland, lors de leur dernière discussion dans le Passage avant leur séparation, qui vont venir redoubler la scène du manège, c'est-à-dire celle des adieux entre Cécile et Frankie - ce mouvement fait quelque peu perdre au spectateur ses repères dans les déplacements des personnages dans le Passage, et plus généralement ce mouvement modifie l'aspect $\mathrm{du}$ Passage. Cependant, comme le souligne Suzanne Liandrat-Guigues, les transformations apparentes du Passage ne doivent rien à l'art du maquillage, et, à travers les mouvements de caméra, elles ne sont que "le fait de la flânerie qui fonctionne comme un opérateur de métamorphose $\aleph^{18}$. Autrement dit, Demy se contente d'habiter le Passage au moyen de sa caméra, c'est-à-dire de répéter, dans le médium cinématographique, le mouvement de la flânerie, dans une architecture ellemême non transformée en décor. Cela dit, les virtualités du passage Pommeraye ne sont probablement pas révélées de la manière la plus probante dans ce premier longmétrage de Jacques Demy, ne serait-ce que parce qu'initialement, ce film (qui aurait dû s'appeler Un billet pour Johannesburg) devait être chanté, et en couleurs, ce qui aurait évidemment donné au Passage, par contraste, un aspect plus éclatant de réalité matérielle, puisque dans le film effectivement réalisé, Lola, la ville est filmée en noir et blanc, de façon réaliste, donc, et les dialogues sont parlés. Il y a par conséquent une forme de continuité qui se dessine entre les scènes hors du Passage et les scènes dans le Passage, déjà du point de vue de la continuité de l'intrigue : si la rencontre entre Roland et Lola constitue bien une rupture dans le récit, elle demeure homogène au temps global de la fiction, en ce qu'elle ne constitue pas l'irruption d'une temporalité hétérogène. Or, un tel surgissement n'aurait été possible, qu'à la condition que ce soit la temporalité propre à l'appareil constitué par le Passage comme architecture urbaine spécifique qui surgisse comme événement. Un tel surgissement de l'appareillage photographique est d'ailleurs effectué, dans le film Model Shop (1968), lorsque George, regardant chez lui les photos en noir et blanc de Lola, qu'il a lui-même prises dans la «boutique » où elle pose pour ce genre de photos un peu déshabillées, voit subitement surgir une image en mouvement, cinématographique, donc, et en couleurs, de Lola - et 
cette irruption à l'écran ne dure qu'un instant. Cela suffit pour opérer une rupture temporelle, et créer un écart entre le personnage de Lola, filmé, et celui de Lola, donné à voir à travers la photo - Demy filmant une photo, il est contraint de recourir à cette trouvaille (la transfiguration de l'image fixe en noir et blanc en image mobile en couleurs) pour donner à voir cette image spécifiquement photographique (que George a entre les mains), et non pas cinématographique. La dimension mentale de l'image mouvante et en couleurs ne fait pas de doute, et la photographie est donc ainsi réduite à l'état de ruine, ce qui la rend visible comme photo.

11 Dans le cadre du premier long-métrage de Demy, Lola, on peut donc dire qu'appareil cinématographique et appareil du passage s'entre-répondent, même si cela s'effectue largement aux conditions de l'appareil cinématographique, c'est-à-dire que cette entreexpression conserve au film sa structure monadique, empêchant le surgissement d'une extériorité - celle de l'appareil urbain des passages, qui est ici davantage mis en scène que seulement montré. Il faudrait donc que l'appareil cinématographique accepte d'abandonner l'hégémonie (temporelle, homogénéisante) du récit intégrant le Passage comme un de ses éléments, pour que l'espace du passage puisse véritablement surgir. C'est précisément parce que le cinéma ne reproduira pas l'espace du passage tel qu'il pouvait fonctionner au $\mathrm{XIX}^{\mathrm{e}}$ siècle, même à travers une image adaptée au $\mathrm{Xx}^{\mathrm{e}}$ siècle, permettant de l'intégrer au tissu narratif - et même si cette image est conforme à celle du passage Pommeraye de l'enfance de Jacques Demy, où il acheta sa première caméra de cinéma- qu'il rendra possible son surgissement dans le cadre de l'appareil cinématographique. Cela réclame donc qu'au lieu de rechercher une entre-expression entre ces deux modes d'appareillage, on essaie d'enrayer ce jeu de miroir, de rompre la continuité temporelle.

\section{Les Parapluies de Cherbourg (1963) - un Passage remémoré}

C'est par conséquent plutôt du côté des Parapluies de Cherbourg qu'il faut se tourner, et plus précisément du côté de la scène du dîner lors duquel Roland Cassard va demander la main de Geneviève (Catherine Deneuve) à sa mère. De fait, comme un effet de rupture dans la temporalité des Parapluies de Cherbourg, une extériorité (cinématographique d'abord, puis bientôt architecturale) perce, au moment où le thème musical de Lola revient, faisant ainsi surgir des images du passage Pommeraye le montrant désert, statufié, comme une manière d'indiquer la disparition de celle qui enchantait le lieu pour Roland Cassard. Les fantasmagories constitutives du fétichisme de la marchandise - au nombre desquelles l'attrait sexuel pour les «créatures des passages »- disparaissent métaphoriquement ici (c'est la fin de l'histoire d'amour de Roland qui a désenchanté le lieu, du point de vue de la fiction), mais ce phénomène renvoie plus profondément à une substitution d'appareil: c'est l'appareil cinématographique en tant que tel, c'est-à-dire en deçà du cadre narratif du film, qui va ici imposer au passage Pommeraye sa temporalité, aboutissant à en faire un lieu inerte, sans vie, autant dire une ruine - condition pour la conduire à sa signification, c'est-àdire ici, à sa manifestation comme architecture, au sein de l'appareil cinématographique. Le travail de mortification des passages est ici vraiment entamé.

Le Passage où s'enchâssent les vies successives comme les choses accumulées est devenu cosa mentale [écrit Suzanne Liandrat-Guigues]. Règnent seulement le décor, 
l'espace immobile, surchargé, délié de toute action et le sentiment de déréliction de cette peinture du vide. Le regard de la caméra coïncide avec une désappropriation de la perception ${ }^{19}$. " corrélations ordinaires de la vie ", ce lieu est rendu à sa vocation de nature morte, il est réduit à l'état de ruine. En cela, le travail de l'allégoriste est réussi, ayant brisé cet objet (au moyen du départ de Lola, qui a retiré à ce lieu tout son sang pour Roland, qui développe cette vision du Passage), mais ce travail en arrive cette fois à se dédoubler, les ruines étant en effet conservées, sous leur aspect mortifère, laissant percer la dimension technique sous le recouvrement artistique - c'est que la rareté (le vide) dénude les ornements, qui n'étaient vivants qu'à redoubler la profusion de la marchandise, lesquels laissent désormais percer, ou au moins deviner, l'architecture de fer les sous-tendant, et qui en est «l'inconscient structurel $»^{20}$. Cette mise à nu de l'architecture du Passage libère ainsi le processus de la remémoration, qu'on prendra soin de distinguer du simple souvenir : l'évocation, par Roland, de son amour pour Lola enchaîne sur des images du passage Pommeraye se présentant à son souvenir, et l'aspect désert des lieux est l'image, la métaphore de la désolation des lieux lorsque ceux-ci ne sont plus animés du souffle de cet amour, et sont donc devenus incapables de produire des images de rêve ; mais pour le spectateur, qui éventuellement d'ailleurs n'a pas vu le film Lola, c'est une forme architecturale dévitalisée qui apparaît à l'écran, incapable de produire la moindre image onirique - et c'est bien là que joue le phénomène de la remémoration: non pas dans la logique narrative de la présence fantomatique de Lola, dans l'esprit de Roland, livré à ses souvenirs volontaires, puisque pour lui les liens du Passage avec sa vie restent vivaces, ce qui l'empêche de faire de ce lieu, même désenchanté, une simple ruine, mais dans le spectacle objectivé sur l'écran du Passage déserté, mettant en évidence la dimension architecturale du lieu, autant dire sa dimension inconsciente, celle qu'on ne pouvait percevoir aussi longtemps que des corrélations vitales nous reliaient à ce lieu. C'est en ce sens alors qu'on pourrait accepter l'idée que le cinéma de Demy est un cinéma de rêve. En cela, on retrouverait la position de Kracauer, lorsqu'il écrit :

On peut à bon droit dire des films vraiment cinématographiques qu'ils ressemblent par moments à du rêve; mais ces moments n'ont rien à voir avec ceux où ces films s'évadent au royaume de l'imaginaire et de la fantasmagorie mentale. Les plus oniriques sont au contraire ceux où la caméra se concentre sur les phénomènes de la vie réelle. [...] Peut-être que le film se rapproche le plus du rêve quand il nous submerge sans ménagement sous la présence crue d'objets naturels - comme si la caméra venait tout juste de les extirper de la matrice de l'existant matériel et que le cordon ombilical entre l'image et la réalité présente n'avait pas encore été tranché. Il y a dans l'immédiateté abrupte et dans la véracité choquante de telles images quelque chose qui justifie qu'on les assimile à des images de rêve ${ }^{21}$.

Il ne faut bien évidemment pas entendre, à travers l'idée d'un lien non encore rompu « entre l'image et la réalité présente » évoquée par Kracauer, qu'il aurait ici en vue la saisie de l'existence sous son aspect nécessairement le plus contemporain, car cette réalité serait alors notre réalité vécue, et serait donc inapte à provoquer le moindre choc, incapable alors d'être identifiée à la moindre «image de rêve"-c'est le surgissement de la matérialité du monde sur l'écran qui en fait toute l'actualité, et non la dimension vécue que la chose, en sa dimension référentielle, conserverait avec notre propre existence présente. Il ne s'agit donc pas de capter la réalité, dans une vision naïvement positiviste du cinéma, mais de la conduire à se révéler sur l'écran, comme on 
parle d'un révélateur photographique, c'est-à-dire d'un produit capable de conduire l'image à sa visibilité. "L'art ne représente pas, il rend visible », soutenait Paul Klee, et c'est bien en ce sens qu'il faut entendre les mots de Kracauer, fidèles en cela à la pensée de Benjamin, lui-même en écho évident avec Klee. Il reste à envisager à présent une dernière référence au passage Pommeraye dans l'œuvre cinématographique de Demy, dans le cadre du film Une chambre en ville, une dimension supplémentaire intervenant ici, du moins de façon plus explicite, relevant du rapport entre la rue et le passage même s'il est bien certain que cette dimension de communication entre la rue et l'extérieur n'était pas absente auparavant, ne serait-ce qu'à travers les entrées et sorties des personnages.

\section{Une chambre en ville (1982) - la rue et le Passage}

15 On peut d'abord faire remarquer que dans Une chambre en ville, le Passage apparait véritablement comme "hors d'usage » et "hors temps " (on n'aperçoit le Passage que désert, éclairé mais sans présence de marchandises, sinon, les postes de télévision, éternellement allumés, dans la boutique d'Edmond; si, d'autre part, Edith joue le rôle de la demi-prostituée, elle est surtout une bourgeoise malheureuse dans son existence conjugale, qui peut à l'occasion se prostituer gratuitement pour qui lui plait). Cette présentation du Passage comme littéralement désaffecté (déserté, mais aussi privé d'affects) vient renforcer le processus qui est déjà largement entamé dans Les Parapluies de Cherbourg:

[La] simple exposition [du Passage] supplée peu à peu à tout étalage commercial, à toute image de la réalité pour que triomphe sa propre évidence élevée à la dimension d'une nature morte ${ }^{22}$.

Cependant, un élément d'importance est à prendre en considération à propos de ce film, et qui est le fait qu'aux personnages individuels vient s'ajouter cette fois la masse des ouvriers métallurgistes en révolte. Or, si la fantasmagorie est bien une rêverie collective, comme le souligne Benjamin, ce n'est que pour l'individu que l'architecture est extérieure, le collectif, lui, absorbant cette architecture, comme il le fait avec le spectacle cinématographique d'ailleurs. Là où l'individu se déplaçant dans le passage urbain sera à l'intérieur de celui-ci, pour le collectif, il en ira par conséquent tout autrement, le passage constituant pour la figure du flâneur un phénomène intérieur, comparable aux phénomènes kinesthésiques (ou de proprioception) pour l'individu. Dans ces conditions, le film Une chambre en ville, au-delà même des intentions de Jacques Demy, qui visait certes à réaliser un film témoignant des luttes ouvrières dans la région de Nantes et de Saint-Nazaire, permet de poser la question de la dimension politique du réveil, puisque si réveil politique il y a, selon cette logique benjaminienne de la perception, il ne peut relever, on l'a dit, de la prise de conscience, qui réintroduirait une coupure entre rêve et veille. C'est le phénomène de la remémoration qui est politique - ce qui implique que la « faible force messianique » que le passé fait peser sur chaque génération, dont parlent les "Thèses » sur l'histoire, ne peut donc consister à vouloir le retour de ce dont on pourrait prendre conscience, par un simple effort, volontaire, de la mémoire. Ainsi, notre interrogation poursuivie sur la manière dont le passage est rendu présent dans ce film conserve tout son sens, étant entendu que cette révélation continuée du passage Pommeraye s'inscrit alors dans le sillage de la démarche des Parapluies de Cherbourg, de ce point de vue du moins. 
17 On a déjà souligné le fait que le Passage apparaît comme frappé d'inanité dans ce film, comme une chose du passé devenue incompréhensible, à l'image des objets dont le rassemblement objectif semble incohérent. C'est le travail de l'allégoriste qui se poursuit, et comme le souligne encore très justement Suzanne Liandrat-Guigues, constatant que nombre de scènes de rues sont en fait tournées en studio,

[...] comme dans cette peinture hollandaise étrangère aux tumultes de la vie portuaire et à l'exploration d'une société tournée vers ses colonies et son ailleurs, le film de Demy semble vouloir n'offrir que l'intensité d'une peinture d'intérieurs [...] qui élève la ville entière à une méditation sur l'effet de cette introversion ${ }^{23}$.

Si l'on suit cette suggestion, cela signifierait par conséquent que le travail du cinéaste Demy, consistant à rendre visible le passage Pommeraye, et pour cette raison, réclamant qu'il le réduise à l'état de ruine, se serait étendu à l'ensemble de la ville de Nantes selon un procédé tout autre il est vrai : le tournage en studio, par exemple pour un certain nombre des scènes d'affrontement avec les CRS. Le point commun entre la manière de rendre visible le Passage, en le filmant en sa matérialité, donc, mais en le détachant des " corrélations ordinaires de la vie », et le tournage en studio de scènes de rues, ne se comprend qu'à la condition d'envisager le retournement entre intériorité et extériorité, initié à travers le rapport Passage/rue. En effet, si la mortification du Passage réclame qu'on en fasse un élément d'extériorité par rapport à la vie qui traverse la fiction, et qui tend spontanément à s'étendre aux objets et architectures mobilisés par l'action, en revanche, il fallait faire de l'action un élément intérieur, dans le sens où elle devait s'opposer aux extérieurs-rue : la mortification des lieux, étendue à la ville elle-même, risquerait d'être entravée par l'action qui s'y déploierait. Pour éviter la contamination de la ville par la vie de l'action, on tourna en studio. Si la mort est en effet omniprésente dans Une chambre en ville, c'est bien sûr parce que la mort des amants (Édith et François) en constitue le symbole évident, mais c'est aussi, plus profondément, parce que le processus de constitution du cinéma de Demy en allégorie de l'allégorie se poursuit, commandant qu'on déréalise encore un peu plus le passage Pommeraye (au fond, c'est encore par la médiation des appareils de télévision - par procuration, donc - que la vie se manifeste le plus nettement dans le passage, sous la forme des images en mouvement d'un monde lui-même en mouvement, à l'intérieur de la boutique d'Edmond, par conséquent, bien qu'elle symbolise par ailleurs l'enfermement par excellence, sous la forme de la geôle maritale), mais aussi qu'on mortifie le lieu référentiel des luttes pour, par-delà toute perception consciente, en provoquer la remémoration.

Des ouvriers et des CRS qui, respectivement, s'insultent et se menacent en chantant, cela ne peut en effet que provoquer un effet de distance qui, mettant à l'écart toute forme de souvenir conscient de luttes sociales, n'en est pas moins bouleversant pour l'effet souterrain que cette démarche produit, et que le lyrisme de la musique de Michel Colombier renforce. Que le mélodrame qu'est Une chambre en ville joue sur le pathétique, c'est une évidence, et pourtant, il le fait en délaissant les formes classiques d'identification, du fait du peu de réalisme de la réalisation - en cela, sa démarche, répétons-le, s'apparente notamment à celle de Fassbinder. Il faut donc entendre ce recours au pathos en un sens infra-conscient - le spectateur ne comprend pas bien l'émotion qui s'empare de lui, évidente pourtant en ses effets. La cause n'en serait-elle pas ce qu'on n'a pas vécu, et qui pourtant revient? Ne serait-ce pas, selon la belle expression de Françoise Proust, 
l'enfance toujours déjà perdue, [qui] revient et renaît, c'est-à-dire naît enfin à jamais, par bouffées, par éclairs, dans les rires et les larmes, lorsqu'un instant, inattendu, "inouï", suscite et comble la nostalgie d'un passé qui fut heureux, fugitivement, d'un savoir insu ${ }^{24}$ ? Demy dans le cadre de ce qui deviendrait une orthodoxie kracauerienne du cinéma (Kracauer s'est en effet toujours intéressé à des séquences de films, et ne s'est donc guère prononcé quant à la globalité d'une œuvre), on peut suggérer une réserve quant au traitement que Kracauer fait subir à un certain type de place accordée à la musique au cinéma. En effet, même s'il est certain que la forme de cinéma « en chanté » initiée par Demy ne correspond pas à celle des comédies musicales, et que par conséquent, Kracauer ne pouvait anticiper l'originalité de Demy, certaines remarques semblent pouvoir être élargies au cinéma intégralement chanté, sans trop de difficulté. Que dit Kracauer? Remarquons tout d'abord qu'il reconnaît à la musique un rôle essentiel dans le cinéma, dès les premiers films muets, non pas pour souligner la signification des images, mais en vue "d'adapter physiologiquement le spectateur au flux des images ${ }^{26}$ - c'est que dans la réalité, en effet, nous ne voyons guère d'objet sans que des sons accompagnent notre sensation visuelle - le couplage œil/oreille est donc une habitude intégrée à nos modalités perceptives. Par ailleurs, il porte un jugement plutôt positif, cinématographiquement parlant, sur la comédie musicale, arbitrant de façon équilibrée, selon lui, l'opposition éternelle entre la «tendance réaliste » (l'intrigue, souvent fruste) et la «tendance formatrice " (s'exprimant dans les chansons). Or, dans Les Parapluies de Cherbourg et dans Une chambre en ville, on est plus près - surtout dans ce dernier cas, d'ailleurs - de l'opéra, et il se trouve que Kracauer juge que « [l]e monde de l'opéra est construit sur des prémisses qui contredisent radicalement celles de l'approche cinématographique $»^{27}$. Mais chez Demy, il est bien certain qu'on n'a pas un opéra, qu'il s'agirait, ensuite, de porter à l'écran, mais une œuvre, immédiatement envisagée comme cinématographique et chantée.

Dans ces conditions, le film dont parle Kracauer, et qui s'apparenterait le plus à la tentative de Demy, dans la forme du moins, serait The Medium (1951), de Gian Carlo Menotti, que l'auteur juge très sévèrement, considérant qu'il s'agit là d'un « hybride [...] instable », consistant à « faire [...] à la fois un authentique opéra et un authentique film, un véritable opéra pour l'écran ", avec le résultat suivant, selon Kracauer : "Ce n'est pas que nous soyons simplement dérangés par l'étrange artificialité de ces récitatifs ou par leur effet paralysant sur les images. Ce qui se passe vraiment, c'est surtout que nous sommes pris dans un affrontement terrifiant entre le réalisme cinématographique et la magie de l'opéra. Le film de Menotti est une tentative avortée pour intégrer deux modes d'expression qui s'excluent mutuellement pour des raisons historiques, sociales et esthétiques. Et le spectateur ou l'auditeur quelque peu sensible qui assiste à leur fusion contrainte risque fort de se sentir comme écartelé "28. Le "réalisme cinématographique ", ce serait cette "caméra [qui] explore les scènes de rue, les meubles et les expressions de visage avec une curiosité insistante qui emmène l'imagination du spectateur vagabonder dans les immensités de la réalité matérielle ", 
alors que « la magie de l'opéra ", ce serait le fait de "donne[r] aux plus banals propos des personnages la forme d'un récitatif, si bien que le spectateur se mue en auditeur fortement tenté de fermer les yeux $»^{29}$.

Gageons que Jacques Demy aura réussi à mettre en place une forme cinématographique qui, pleinement cinématographique, aura tout de même su, esthétiquement parlant, s'ouvrir aux puissances de l'opéra. Autrement dit, il ne s'est pas agi, pour lui, de chercher "l'union parfaite entre ces deux médias " $^{30}$, le cinéma et l'opéra, mais de laisser le cinéma être altéré par l'irruption d'un souffle opératique - dans le cas d'Une chambre en ville, c'est la puissance épique du film qui gagne à travers cet apport de musique et de récitatifs. À la différence de Gian Carlo Menotti, Demy, qui ne s'est certes pas inscrit dans le courant néo-réaliste, échappe au vérisme dont relèvent les images cinématographiques de l'Italien, et il a opté, dans Une chambre en ville, pour un jeu sobre des acteurs, à distance de l'outrance du jeu théâtralisé de l'opéra (si ce n'est dans le cas de Michel Piccoli, dont le jeu outré vaut - en même temps que référence au réalisme poétique de Carné, et au personnage joué par Pierre Brasseur dans Les Portes de la nuit comme une citation des formes opératiques du jeu d'acteur, à l'instar d'ailleurs du suicide d'Edith, s'écroulant sur le corps de François). Au fond, Demy a mêlé constamment réalisme et magie, au point d'en brouiller les frontières, et c'est à l'intérieur de ce jeu que doit se comprendre la contamination de son cinéma par la musique et le chant. L'indice le plus sûr que le hiatus entre réalisme et magie est évité, notamment dans Une chambre en ville, et que le jugement de Kracauer quant au lien cinéma/opéra se serait peut-être inversé ici, c'est que la réalité des images elle-même, dans le cas des scènes de combat entre manifestants et CRS par exemple, est obtenue à travers des effets de réalité, ces combats, on l'a dit, étant en fait tournés, pour l'essentiel, en studio, alors que la présence matérielle du passage Pommeraye, dans ce film intégralement chanté, est obtenue, elle, par des effets de déréalisation, de mortification, c'est-à-dire en le laissant à sa pure présence architecturale, valant comme nature morte.

\section{BIBLIOGRAPHIE}

Benjamin Walter, Charles Baudelaire. Un poète lyrique à l'apogée du capitalisme, Jean Lacoste (trad.), Paris, Payot, 1979.

Benjamin Walter, Paris, capitale du XIX⿸e siècle, Jean Lacoste (trad.), Paris, Éditions du Cerf, 1989.

Benjamin Walter, «L'œuvre d'art à l'époque de sa reproductibilité technique » (dernière version de 1939), in Euvres III, Maurice de Gandillac (trad.), revue par Rainer Rochlitz, Paris, Gallimard, 2000, p. 269-316.

Benjamin Walter, «Sur le concept d'histoire », in Euvres III, Maurice de Gandillac (trad.), revue par Pierre Rusch, Paris, Gallimard, 2000, p. 425-443.

Berthomé Jean-Pierre, Jacques Demy et les racines du rêve, Nantes, L'Atalante, 1996. 
Déotte Jean-Louis, « Les appareils urbains », in Spielraum : W. Benjamin et l'architecture, Libero Andreotti (dir.), Paris, Éditions de La Villette, 2011, p. 73-85.

Déotte Jean-Louis, Walter Benjamin et la forme plastique, Paris, L’Harmattan, 2012.

Kracauer Siegfried, Théorie du film. La rédemption de la réalité matérielle, Paris, Flammarion, 2010.

Liandrat-Guigues Suzanne, Modernes flâneries du cinéma, De l'incidence Éditeur, 2009.

Meschonnic Henri, «L'allégorie chez Walter Benjamin, une aventure juive », in Walter Benjamin et Paris, Heinz Wisman (dir.), Paris, Éditions du Cerf, 1986, p. 707-742.

Proust Françoise, L'histoire à contretemps. Le temps historique chez Walter Benjamin, Paris, Éditions du Cerf, 1994.

Sierek Karl, « Double conversion : Ophuls à Vienne », 1895. Mille huit cent quatre-vingt-quinze, $\mathrm{n}^{\circ}$ 34-35, 2001, p. 27-48, http://1895.revues.org/166

\section{NOTES}

2. Françoise Proust, L'histoire à contretemps. Le temps historique chez Walter Benjamin, Paris, Éditions du Cerf, 1994, p. 83.

3. Walter Benjamin, «L'œuvre d'art à l'époque de sa reproductibilité technique » (dernière version de 1939), Maurice de Gandillac (trad.), revue par Rainer Rochlitz, in CEuvres III, Paris, Gallimard, 2000, p. 311-313 (c'est Benjamin qui souligne).

4. Jean-Louis Déotte, "Les appareils urbains ", in Spielraum: W. Benjamin et l'architecture, Libero Andreotti (dir.), Paris, Éditions de La Villette, 2011, p. 74-83.

5. Ibid., p. 83.

6. Jean-Pierre Berthomé, Jacques Demy et les racines du rêve, Nantes, L'Atalante, 1996, p. 330.

7. Suzanne Liandrat-Guigues, Modernes flâneries du cinéma, De l'incidence Éditeur, 2009, p. 43.

8. Siegfried Kracauer, Théorie du film. La rédemption de la réalité matérielle, Paris, Flammarion, 2010, p. 13-14 (je souligne).

9. Jean-Louis Déotte, Walter Benjamin et la forme plastique, Paris, L'Harmattan, 2012, p. 10.

10. Karl Sierek, «Double conversion : Ophuls à Vienne », 1895. Mille huit cent quatre-vingt-quinze, $\mathrm{n}^{\circ}$ 34-35, 2001, http://1895.revues.org/166

11. Ibid.

12. Henri Meschonnic, "L'allégorie chez Walter Benjamin, une aventure juive", in Walter Benjamin et Paris, Heinz Wisman (dir.), Paris, Éditions du Cerf, 1986, p. 714.

13. Walter Benjamin, Charles Baudelaire. Un poète lyrique à l'apogée du capitalisme, Jean Lacoste (trad.), Paris, Payot, 1979, p. 227.

14. Walter Benjamin, Paris, capitale du XIX siècle, p. 73.

15. Suzanne Liandrat-Guigues, Modernes flâneries du cinéma, p. 33-34.

16. Jean-Louis Déotte, « Les appareils urbains », p. 78.

17. Suzanne Liandrat-Guigues, Modernes flâneries du cinéma, p. 34.

18. Ibid., p. 37.

19. Ibid., p. 40.

20. Jean-Louis Déotte, Walter Benjamin et la forme plastique, p. 20.

21. Siegfried Kracauer, Théorie du film. La rédemption de la réalité matérielle, p. 244-245 (je souligne).

22. Suzanne Liandrat-Guigues, Modernes flâneries du cinéma, p. 41-43.

23. Ibid., p. 41.

24. Françoise Proust, L'histoire à contretemps. Le temps historique chez Walter Benjamin, p. 84 . 
25. Walter Benjamin, «Sur le concept d'histoire ", in Euvres III, Maurice de Gandillac (trad.), revue par Pierre Rusch, Paris, Gallimard, 2000, p. 428.

26. Siegfried Kracauer, Théorie du film. La rédemption de la réalité matérielle, p. 203.

27. Ibid., p. 230.

28. Ibid., p. 231-232.

29. Ibid., p. 232.

30. Ibid., p. 231.

1. Walter Benjamin, Paris, capitale du XIX ${ }^{e}$ siècle, Jean Lacoste (trad.), Paris, Éditions du Cerf, 1989.

\section{RÉSUMÉS}

À l'origine de ce texte, il y a le constat que les lieux propres au cinéma de Jacques Demy entretiennent une indéniable proximité avec ceux que privilégie le livre inachevé de Walter Benjamin, Paris, capitale du XIX siècle $e^{1}$ en l'occurrence, c'est le passage Pommeraye, situé dans la ville de naissance du cinéaste, qui retiendra toute notre attention. À partir de l'idée selon laquelle les passages urbains et le cinéma constituent deux formes spécifiques d'appareillage de notre perception, il s'agit alors d'interroger les rapports, évoluant à travers l'œuvre, qui se développent entre ces deux formes d'appareil, sur le fond de cette communauté perceptive des spectateurs d'architecture et de cinéma, que signalait Benjamin. On verra que les interventions du passage Pommeraye, dans les films de Jacques Demy, se révèlent probablement les plus intéressantes lorsque la caméra, n'en faisant pas un élément de décor, parvient à en révéler toute la matérialité - et ce, d'autant mieux que le cinéaste parviendra, paradoxalement, à mortifier ce lieu. En cela, Demy renouvellerait, sur le plan cinématographique cette fois, la pratique de l'allégoriste, telle que Benjamin l'envisageait, tout en prolongeant les analyses de Kracauer quant aux rapports entre cinéma et matérialité.

\section{INDEX}

Mots-clés : architecture, cinéma, fantasmagorie, le flâneur, matérialité, passage Pommeraye, perception, remémoration, rêve, réveil

Personnes citées : Demy (Jacques), Kracauer (Siegfried), Ophuls (Max), Benjamin (Walter) 